\title{
$\angle S$ Research Square \\ Tracking genetic diversity of SARS-CoV-2 infections in Ghana after one year of surveillance
}

\section{Collins Morang'a}

West African Centre for Cell Biology of Infectious Pathogens (WACCBIP)

Joyce Ngoi

Centre for Geographic Medicine Research (Coast), Kenya Medical Research Institute-Wellcome Trust

Research Programme

Jones Gyamfi

University of Health and Allied Sciences COVID-19 Testing and Research Centre

Dominic Amuzu

West African Centre for Cell Biology of Infectious Pathogens (WACCBIP)

Benjamin Nuertey

Tamale Teaching Hospital Intensive Care Unit

Philip Soglo

West African Centre for Cell Biology of Infectious Pathogens (WACCBIP)

Vincent Appiah

West African Centre for Cell Biology of Infectious Pathogens (WACCBIP)

Ivy Asante

Noguchi Memorial Institute for Medical Research

Paul Owusu-Oduro

LEDing Medical Laboratory

\section{Samuel Armoo}

Biomedical and Public Health Research Unit

\section{Dennis Adu-Gyasi}

Kintampo Health Research Centre

\section{Nicholas Amoako}

Kintampo Health Research Centre

\section{Joseph Oliver-Commey}

Ghana Infectious Disease Centre

\section{Michael Owusu}

Kumasi Centre for Collaborative Research in Tropical Medicine

\section{Augustina Sylverken}

Kumasi Centre for Collaborative Research in Tropical Medicine

\section{Edward Fenteng}

Accra Veterinary Laboratory 


\section{Violette M'cormack}

West African Centre for Cell Biology of Infectious Pathogens (WACCBIP),

\section{Frederick Tei-Maya}

West African Centre for Cell Biology of Infectious Pathogens (WACCBIP), https://orcid.org/0000-00034507-1788

\section{Evelyn Quansah}

West African Centre for Cell Biology of Infectious Pathogens (WACCBIP),

\section{Reuben Ayivor-Djanie}

University of Health and Allied Sciences COVID-19 Testing and Research Centre

\section{Enock Amoako}

West African Centre for Cell Biology of Infectious Pathogens (WACCBIP)

\section{Isaac Ogbe}

West African Centre for Cell Biology of Infectious Pathogens (WACCBIP)

\section{Bright Yemi}

West African Centre for Cell Biology of Infectious Pathogens (WACCBIP)

\section{Israel Osei-Wusu}

West African Centre for Cell Biology of Infectious Pathogens (WACCBIP)

\section{Deborah Mettle}

West African Centre for Cell Biology of Infectious Pathogens (WACCBIP)

\section{Samirah Saiid}

West African Centre for Cell Biology of Infectious Pathogens (WACCBIP)

\section{Kesego Tapela}

West African Centre for Cell Biology of Infectious Pathogens (WACCBIP)

\section{Francis Dzabeng}

West African Centre for Cell Biology of Infectious Pathogens (WACCBIP)

\section{Vanessa Magnussen}

Noguchi Memorial Institute for Medical Research

\section{Jerry Quaye}

West African Centre for Cell Biology of Infectious Pathogens (WACCBIP)

\section{Precious Opurum}

West African Centre for Cell Biology of Infectious Pathogens (WACCBIP)

\section{Rosina Carr}

University of Health and Allied Sciences COVID-19 Testing and Research Centre

\section{Patrick Ababio}

Accra Veterinary Laboratory

\section{Abdul-Karim Abass}

Tamale Public Health and Reference Laboratory

\section{Samuel Akoriyea}

Institutional Care Division (ICD) 


\section{Emmanuella Amoako}

Cape Coast Teaching Hospita

\section{Frederick Kumi-Ansah}

Cape Coast Teaching Hospita

\section{Oliver Boakye}

Takoradi Veterinary Services Department

\section{Dam Mibut}

Tamale Teaching Hospital Intensive Care Unit

\section{Theophilus Odoom}

Takoradi Veterinary Services Department

\section{Lawrence Ofori-Boadu}

Ga East Municipal Hospital

\section{Emmanuel Allegye-Cudjoe}

Pong-Tamale Central Veterinary Laboratory

\section{Sylvester Dassah}

Navrongo Health Research Centre

\section{Victor Asoala}

Navrongo Health Research Centre

\section{Kwaku Poku Asante}

Kintampo Health Research Centre

\section{Richard Phillips}

Kumasi Centre for Collaborative Research in Tropical Medicine

\section{Mike Osei-Atweneboana}

Biomedical and Public Health Research Unit

\section{John Gyapong}

University of Health and Allied Sciences COVID-19 Testing and Research Centre

\section{Patrick Kuma-Aboagye}

Ghana Health Service

\section{William Ampofo}

Noguchi Memorial Institute for Medical Research

\section{Kwabena Duedu}

University of Health and Allied Sciences COVID-19 Testing and Research Centre

\section{Nicaise Ndam}

Université de Paris

\section{Yaw Bediako}

West African Centre for Cell Biology of Infectious Pathogens (WACCBIP)

\section{Peter Quashie}

West African Centre for Cell Biology of Infectious Pathogens (WACCBIP)

\section{Lucas Amenga-Etego}


West African Centre for Cell Biology of Infectious Pathogens (WACCBIP)

Gordon Awandare ( $\square$ gawandare@ug.edu.gh )

West African Centre for Cell Biology of Infectious Pathogens (WACCBIP)

\section{Article}

Keywords: SARS-CoV-2, molecular evolution, spatio-temporal dynamics

Posted Date: November 23rd, 2021

DOI: https://doi.org/10.21203/rs.3.rs-1088719/v1

License: (c) (i) This work is licensed under a Creative Commons Attribution 4.0 International License. Read Full License

Version of Record: A version of this preprint was published at Nature Communications on May 6th, 2022. See the published version at https://doi.org/10.1038/s41467-022-30219-5. 


\section{Abstract}

This study sequenced 1077 SARS-CoV-2 genomes from patient isolates (106 from arriving travellers and 971 from communities) to track the molecular evolution and spatio-temporal dynamics of the SARS-CoV2 variants in Ghana. The data show that initial local transmission was dominated by B.1.1 lineages, but the second wave in Ghana was overwhelmingly driven by the Alpha variant, which was detected in community cases from January 2021, with Eta also contributing to reported cases. Subsequently, an unheralded variant under monitoring, B.1.1.318, dominated transmission from April to June 2021 before being displaced by Delta (B.1.1.617) and Delta Plus (AY.*) variants, which were introduced into community transmission in May 2021 and have remained dominant to date. Mutational analysis indicated that variants that took hold in Ghana harboured transmission enhancing and immune escape spike substitutions. The apparent rapid viral evolution observed demonstrate the potential for emergence of novel variants with greater mutational fitness.

\section{Introduction}

A year after the World Health Organization (WHO) declared the coronavirus disease 2019 (COVID-19) caused by severe acute respiratory syndrome coronavirus 2 (SARS-CoV-2) a pandemic, over 190 million confirmed cases and 4 million deaths have been reported worldwide ${ }^{1}$. As of 27 th September 2021, Ghana's cumulative COVID-19 cases stood at 127482 with an active case count of 3088 and 1156 deaths ${ }^{2}$. Through the COVAX initiative, 1.23 million people in Ghana have received vaccine doses, with 376000 fully vaccinated with the AstraZeneca vaccine ${ }^{3,4}$ Compliance to prescribed preventive measures have been low to average within the communities, and there is evidence of persistent albeit mostly asymptomatic infections, especially in Accra and other major cities in Ghana ${ }^{5}$.

COVID-19 control measures in Ghana have evolved with the global pandemic. Ghana's international airports and all land borders were closed to international travel on 22nd March 2020, followed by a partial lockdown of two major cities from March 30th to 22nd April 2020. This was coupled with enhanced testing and contact tracing to track community spread. The airport was reopened to international travel on 1 st September 2020, with two-fold containment measures; (i) travellers must show proof of a negative COVID-19 test (taken at most 72 hours before arrival) and (ii) travellers must be negative for the SARSCoV-2 antigen test upon arrival at the Kotoka International Airport (KIA ${ }^{2}$. The guidelines for travellers who test positive at the airport have changed over time. Initially, travellers who tested positive upon arrival had to undergo mandatory isolation for at least 14 days (at travellers' cost) and were only allowed to go after a negative PCR/antigen test. The guidelines were later relaxed to allow self-isolation, but mandatory isolation has been reinstated due to poor compliance ${ }^{2}$. Currently, a positive test leads to a minimum of 3 days in isolation. After three days, travellers who test negative by RT-PCR after three days are allowed to leave isolation. Since January 2021, all positive samples from travellers have been made available for genomic sequencing. 
Like other RNA viruses, most mutations in the SARS-CoV-2 genome arise naturally and spontaneously during viral replication, transmission, and adaptation cycles in the population. Whole-genome sequencing is critical in tracking viral genomic changes and may help to understand phenotypic changes. According to the WHO, several variants of interest (VOIs) have been shown to harbour genomic mutations associated with enhanced community transmission or multiple COVID-19 cases/clusters in numerous countries ${ }^{6}$. Other VOIs have proven to be variants of concern (VOCs) due to their increased transmissibility, virulence, and disease severity, or decreased susceptibility to public health and social measures, available diagnostics, vaccines, and therapeutics ${ }^{7}$. These may be demonstrated by increased receptor binding, reduced virus neutralisation by antibodies generated against previous infection or vaccination, loss or reduced diagnostic detection, or increased replication (WHO, 2021a). The SARS-CoV-2 VOCs and VOls listed by WHO include; Alpha (B.1.1.7), Beta (B.1.351), Gamma (P.1), Delta (B.1.617.2), Epsilon (B.1.427/B.1.429), Zeta (P.2), Eta (B.1.525), Theta (P.3), lota (B.1.526) and Kappa (B.1.617.1) (WHO, 2021b). These variants were first documented by the UK, Brazil, India, USA, and Philippines ${ }^{6}$. Only Beta and Eta were first reported in South Africa and Nigeria, respectively 6,8 .

Having established a local capacity for sequencing and analysing SARS-CoV-2 genomes in Ghana 9 , molecular surveillance has continued using samples provided by the COVID-19 testing laboratories across the country. In addition, some samples from international travellers who tested positive on arrival at the airport were also analysed to track the introduction of new variants into the country. Thus, this report provides a comprehensive analysis of the genetic diversity of SARS-CoV-2 viruses that caused infections in the communities in Ghana from June 2020 to September 2021.

\section{Results}

\section{Genomic epidemiology of variants circulating in Ghana}

A total of 1077 COVID-19 PCR positive samples from ten regions in Ghana between June 2020 and September 2021 were successfully sequenced. Of these, $44.4 \%$ (479/1077) were near full-length genomes (missingness $(\mathrm{N})<500), 47.8 \%(515 / 1077)$ had 500-4000 unresolved nucleotide assignments, and only $7.7 \%(83 / 1077)$ had 4000-8913 missingness $(\mathrm{N})$. Of 1077 high quality samples, sampling contribution from the different regions in Ghana, was as follows: Ashanti ( $4.4 \%, \mathrm{n}=47)$, Bono East ( $1.2 \%$, $\mathrm{n}=13)$, Central $(11.9 \%, \mathrm{n}=129)$, Eastern $(4.8 \%, \mathrm{n}=52)$, Greater Accra $(43.2 \%, \mathrm{n}=465)$, Northern $(5.1 \%$, $\mathrm{n}=55)$, Upper East $(0.4 \%, \mathrm{n}=4)$, Upper West $(1.1 \%, \mathrm{n}=12)$, Volta $(9.6 \%, \mathrm{n}=103)$ and Western $(8.4 \%, \mathrm{n}=91)$ (Fig. 1a). With the highest number of active COVID-19 cases (GHS, 2021), the Greater Accra region had the highest number of sequenced samples. Only $15.7 \%(169 / 1077)$ of all the samples were collected in 2020, while the remaining were collected in 2021 ( $n=908 / 1077)$. Of the 908 samples collected in 2021, $11.7 \%(n=106)$ were from travellers arriving in the country through the Kotoka International Airport.

Overall, the Alpha (20.8 \%, 224/1077), Delta Plus (18.7\%, 201/1077), B.1.1.318 (16.7\%, 180/1077), Delta (14.8 \%, 159/1077), B.1.1 (9.0 \%, 97/1077), and Eta (4.4\%, 47/1077) made up the top viral lineages within the sequenced SARS-CoV-2 genomes in Ghana over the period (Fig. 1b). Since the B.1.1.318 had 
the third-highest frequency, it is considered a variant under monitoring in Ghana. In 2020, the Ashanti, Central, Eastern, Greater Accra and Western regions had the highest numbers of confirmed cases of COVID-19 in Ghana. Different regions showed variations in relative frequencies of the variants. Genomes which clustered within the B.1.1 lineage dominated samples from Greater Accra $(68.8 \%, 11 / 16)$, Volta region $(55.6 \%, 10 / 18)$, Western region $(51.2 \%, 22 / 43)$ and Central region $(44.4 \%, 20 / 45)$. B.1.623 $(27.8$

$\%, 5 / 18)$ co-dominated with B.1.1 $(27.8 \%, 5 / 18)$ in the Ashanti region whereas B.1.1.359 $(48.3 \%, 14 / 29)$ dominated alongside B.1.1 (34.5\%, 10/29) in the Eastern region (Fig. 1C).

In 2021, there was a marked shift in the circulating variants and occurrence of regional specific outbreaks, with Eta dominating in Northern and middle belt regions, while B.1.1.318 dominated the major cities. The highest frequencies of Eta variants were observed in the Northern $(23.6 \%, 13 / 55)$, Bono $(30.8 \%, 4 / 13)$ and Eastern $(13 \%, 3 / 23)$ regions. The city of Tamale in the Northern region is the gateway, and central trading hub with Ghana's northern neighbours, whilst the Bono region harbours major interaction routes with Ivory Coast in the western corridor of Ghana. Meanwhile, a third of all the variants detected in 2021 were B.1.1.318 (30\%, 176/802), and Greater Accra, where the capital city and the major international airport are located, had $80 \%$ (140/176) of all the genomes. (Fig. 1d). These data suggest that Eta and B.1.1.318 variants, which dominated transmission in these areas in April-May 2021, could have been introduced through these major land borders. Interestingly, the B.1. 1, B.1.359, B.1.1., and B.1.623 that dominated Ghana in 2020 became supplanted by the modal variants responsible for most transmissions in all the regions. It is worth noting that in regions where more than 50 samples were sequenced in 2021, there was penetration or transmission of all the VOCs, including the Central region, Greater Accra, and Volta Region (Fig. 1d).

\section{Importation of SARS-CoV-2 variants into Ghana by travellers}

One hundred and six of the sequenced samples $(9.8 \%, 106 / 1077)$ were obtained from quarantined travellers identified as COVID-19 positive at the KIA. Of this number, Alpha accounted for $44.3 \%(n=47)$ of the genomes while the other VOCs accounted for lower proportions; Beta $(6.6 \%, n=7)$, Delta $(6.6 \%, n=7)$, and Delta Plus $(0.9 \%, n=1)$ (Fig. 2a). The VOls such as Eta $(4.7 \%, n=5)$, Kappa and the local variantunder-monitoring, B.1.1.318, $3.8 \%(n=4)$ were detected at low proportions. Importantly, the VOC Alpha was identified in travellers entering Ghana from all over the World, including other African countries, in January and March 2021 (Fig. 2b and c). Furthermore, VOCs were detected in travellers from several of Ghana's neighbouring countries, demonstrating that these variants were already in those countries even though not reported or detected (Fig. 2c). In most cases, VOCs and VOls were identified amongst quarantined travellers before their detection within local samples. Travellers from Nigeria, Dubai, and the UK accounted for most detections of Alpha, Beta, Delta, Eta, and Delta-plus variants. Interestingly, the Beta and Kappa variants did not become dominant in Ghana; instead, B.1.1.318, which is likely to have originated from Nigeria, and detected in a traveller from Gabon, became dominant in Ghana.

\section{Temporal trends of SARS-CoV-2 variant detection and frequency}


Ghana was one of the last African countries to detect COVID-19 cases in March 2020, and the waves of COVID-19 in Ghana have lagged slightly behind other African countries and significantly behind the rest of the World (Fig. 3a). Previous work from our group described the viral genome dynamics between March and June 2020, when Ghana was largely closed to international travel (Ngoi et al., 2021). WHO (2021) reported that different variants rose to dominance at different times and during different infection waves across the country (Fig. $3 a$ and $3 b$ ). Variants that cluster closely to B.1.1 were first detected in June $2020(44.4 \%, 4 / 9)$ and peaked in July 2020 (58.7\%, 27/46). The B.1.1 lineage remained dominant in August (59.3 \%, n=16/27), and October 2020 (55.2 \%, n=16/29) but in September 2020, B.1.1.359 (55.6 $\%, n=10 / 18)$ became dominant while in December 2020 B.1.623 (33.3 \%, $n=9 / 27)$ was dominant alongside B.1.1 $(29.6 \%, n=8 / 27)$. The Alpha VOC, first detected in local samples from $1^{\text {st }}$ January 2021, quickly supplanted the B.1.1 lineage as the most dominant circulating lineage $(72.3 \%, n=102 / 141)$ in January. Alpha maintained high frequency $(60.0 \%, \mathrm{n}=42 / 70)$ in February 2021 , followed by the VOI, Eta $(20.0 \%, n=14 / 70)$ (Fig. 3b \& 3c). Alpha was still dominant in the March samples $(70.9 \%, 22 / 31)$ but was almost completely supplanted in April 2021, as B.1.1.318 rose to prominence. Sequences clustering to B.1.1.318 had been detected as early as February $2021(4.3 \%, n=3 / 70)$ but became dominant in April $2021(63.3 \%, n=38 / 60)$ alongside Eta $(23.3 \%, n=14 / 60)$. May 2021 represented the peak of B.1.1.318 $(82.3 \%, n=79 / 96)$ and the emergence of the Delta $(3.1 \%, n=3 / 96)$ and Delta Plus $(2.1 \%, n=2 / 96)$ variants in local circulation. Delta and Delta-plus variants increased in proportion over time, overtaking the B.1.1.318 in June 2020. Delta-plus variants surged in July and now dominate all samples, with Delta being the only other variant with a significant presence in Ghana as of September 2021 (Fig. 3c).

\section{Clinical presentation of the COVID-19 cases}

Although most of the samples were from asymptomatic individuals who reported for COVID-19 testing, a good number of genomes (121/971) were from individuals who presented at treatment centres with $\mathrm{mild} /$ moderate or severe/critical symptoms. Majority of the individuals classified as mild/moderate (69.4 $\%, 84 / 121$ ) presented with fever/chills, cough, pains, sore throat, diarrhoea, runny nose, nausea/vomiting, loss of smell, loss of taste or headache. Our data shows that less than a third of individuals presenting at the hospital were classified as severe/critical $(30.5 \%, 37 / 121)$ with difficulty breathing (shortness of breath), hypoxia or multiorgan system dysfunction. The variant type was significantly associated clinical presentations (Pearson chi-square test, $\left.X^{2}=23.4, \mathrm{df}=5, P<.001\right)$, with all the cases of Alpha (100\%, 16/16), as well as the vast majority of patients with Eta (10/11, $90.9 \%)$ and B.1.1.318 (10/11, $90.9 \%)$, presenting with mild/moderate symptoms. On the contrary, about half of cases infected with Delta (20/41, $48.8 \%)$ and Delta-plus (10/19, 52.6 \%) variants exhibited severe/critical COVID-19 (Fig. 3d).

\section{Genetic diversity and evolutionary relationships of the SARS-CoV-2 variants}

Amongst the many individual lineages represented in the data presented here, Delta, Delta Plus, Alpha, B.1.1.318, B.1.1.359, B.1.1, and Eta were the most evolved, with the highest genetic diversity (Fig. 4a). These variants exhibited a local variation in the number of mutations from sample to sample, with Delta, Alpha and B.1.1.318 presenting a mean $~ 30$ (spread/range of 20-45) mutations in the majority of the 
genomes (Fig. 4a). The VOC delta plus, a subline of the Delta, had the highest mean ( 35) and presented a range of mutations from 25 to 45 mutations across all the samples (Fig. 4a). It is worth noting that this level of genetic diversity in the 200 Delta plus samples mainly was attributed to the AY.39 (174/200) and AY.37 (15/200) lineages. Most of the other lineages with a small range of mutations were reported in 2020 and occurred spontaneously in very few samples hence the relatively low genetic diversity (Fig. 4a). The high level of genetic diversity in most VOCs, including the B.1.1318, is probably indicative of Ghana's local evolution and consequential adaptation compared to the other variants that did not gain prominence in the Ghanaian population (Fig. 4a).

A snapshot of the evolutionary relationship of these VOCs in Ghana shows an exciting relationship of variants through space and time throughout the epidemic (Fig. 4b). Using a phylogenetic tree, we outline the transmission events, how the VOCs were introduced, and how they gained prominence coinciding with the COVID-19 waves in Ghana. The outbreak of the COVID-19 pandemic started in mid-November 2019, but then the tree shows that the earliest lineages in Ghana are dated June 2020, although most VOCs were introduced in 2021 (Fig. 4b). The phylogenetic analysis of the genomes from Ghana shows similarities to VOCs around the World, with all the VOCs having the same common ancestor (Wuhan). Still, as they diverge, they share uniquely more recent ancestors; for example, we show that the recently classified sub-lineages of Delta share several recent ancestors in the same clade (Fig. 4b). The root-to-tip divergence of the VOCs as a function of sampling time show a molecular clock of the various VOCs, and with strong evidence, the variants are evolving in a clocklike manner $\left(R^{2}=0.68\right)$ (Fig. $\left.4 \mathrm{c}\right)$. The variants in Ghana are gaining 26.54 mutations per year, and of particular interest is the B.1.1.318 that did not gain prominence worldwide, but its molecular clock is similar to most of the VOCs in Ghana (Fig. 4c). Mutational fitness of the B.1.1.318 lineage showed that ten samples had spike mutations that were likely to confer viral fitness (mutational fitness $>1$ ) (Fig. 4c).

\section{Mutational analysis of the amino acid substitutions}

The most abundant mutation in all the samples was the Spike D614G $(98 \%, 957 / 971)$, followed by ORF1b: P314L (92\%, 900/971) (Fig. 5a). For many of the genes, one or more mutations were occurred in more than 100 samples, although spike protein dominated the profile (Fig. 5a). Interestingly, some variants with different evolutionary lineages had similar amino acid substitutions, mainly spike glycoprotein. The Eta variant had the highest (three) individual mutations (Q52R, Q677H and F888L) in the spike protein compared to other VOCs, contributing to its adaptability in Africa. Compared to other VOCs, the mutations unique to the Alpha variant were S13I, R567K, A570D, and T716I. The only mutation unique to the B.1.1.318 on the spike protein was the D1127G compared to other VOCs and VOIs. (Fig. 5b). Within these samples, the Delta and Delta plus shared fourteen mutations (T19R, G142D, R158G, A222V, L452R, T478K, E484Q, D614G, S680F, P681R, D950N, K1191N, G1219V and C1253F), but the Delta plus had P26L as a unique mutation (Fig. 5b). The unique mutations were fewer than shared mutations, thus explaining the increased abundance of some mutations among the VOCs. Those with the highest frequency in the spike protein among Delta plus, Delta, B.1, B.1.1, B.1.1.318, Alpha, Beta and Eta were fitness substitutions D614G and P681R/H (Fig. 5b). Alpha and B.1.1.318 had the P681H substitution 
while P681R was present in Delta, Eta and Delta-plus variants. Immune escape mutation E484K was present in B.1.1.318, Beta, and Eta, while Delta-plus variants frequently presented with E484Q mutation.

\section{Discussion}

Having established local capacity to generate high-quality genome sequences and comprehensively analyze them in-house, we conducted genomic surveillance in Ghana from June 2020 to September 2021 and performed an in-depth analysis on the resulting 1077 SARS-CoV-2 sequences obtained. This study represents the most extensive genomic analysis of the SARS-CoV-2 viruses driving the COVID-19 pandemic in Ghana. Trends in SARS-CoV-2 infections in Ghana have followed a similar pattern as the rest of Africa and globally, although Ghana has constantly lagged behind the rest of Africa and the World in the COVID-19 disease waves. The emergence of new variants such as Alpha and Delta have been responsible for the second and third COVID-19 disease waves in Ghana, as has been reported in other countries in Africa and globally ${ }^{1}$. The Ghana Health Service monitoring data indicate that the Greater Accra Region was and still is the epicentre of COVID-19 infections ${ }^{2}$. Other regions with major urban cities, including Ashanti, Western and Central, have had high infection levels and similar circulating variants as Greater Accra, likely due to a high volume of intercity travel across these regions. Regions like Northern and Upper East, further from Accra, tended to have different variants during the second wave. In the third wave, these regions still lag behind the rest of the country and do not seem to be undergoing a third wave yet ${ }^{2}$. These regions experience much lower international travellers from global COVID-19 hotspots than Greater Accra and Ashanti. Furthermore, they have a more sparse population with less congested cities than Greater Accra and Ashanti regions. Studies have shown that higher population density increases contact rates necessary for SARS-CoV-2 disease transmission ${ }^{10}$.

Before the airport reopened to international travellers in September 2020, the B.1.1 variant was dominant in Ghana and remained the most dominant circulating lineage throughout 2020. In January 2021, the Alpha variant supplanted B.1.1 and became the leading cause of all reported cases nationally. Our analysis showed that major variants such as Alpha, Beta, Delta, Delta Plus, Eta, and Kappa were detected in samples from arriving travellers before being observed in community cases. This was indicative that VOCs and VOIs were likely imported into Ghana through travellers from other countries, including African countries which had not reported such variants. These results also suggest that the delayed entry of VOCs into Ghana was at least partly due to the mandatory antigen testing on arrival at Kotoka International Airport (introduced when the borders were reopened in September 2020) and subsequent imposition of a policy for compulsory isolation of all antigen-positive passengers (in January 2021). The results also suggest that some individuals escaped detection and seeded local outbreaks. Alpha was the most frequent variant among travellers, followed by the Delta, Beta, Eta, and B.1.1.318, with other variants accounting for the rest. Due to the increased transmissibility of Alpha ${ }^{11}$, it was not surprising that it became the most dominant variant in local transmission in major cities. Eta was primarily responsible for COVID-19 cases that we analyzed from the Northern Region. Eta is likely to have originated in Nigeria but 
was first identified in the UK, and it contains immune escape mutations (del69-70 \& E484K) and increased fitness mutations (P681R) ${ }^{12}$.

Though largely unheralded, B.1.1.318 had been responsible for the second wave in Mauritius ${ }^{13}$. Given the spread of B.1.1.318 in Ghana, Mauritius and over 30 countries, it looked poised to become a dominant SARS-CoV-2 variant. From our dataset, B.1.1.318 appeared to replace Alpha as the predominant lineage in community infections in March 2021 and remained dominant until June 2021, when it was replaced by Delta and Delta plus. The combination of spike substitutions T95I, E484K, D614G, P681H, and D796H in B.1.1.318 may have allowed it to escape Alpha-induced immunity in the Ghanaian population and replace the Alpha variant. This analysis further solidifies the evidence for E484K, L5F, A27S and D614G as spike substitutions that increase infectiousness with minimal impacts on severity ${ }^{14}$. In our study, B.1.1.318, Alpha and Eta were associated with mild/moderate infections, though this interpretation is limited due to the small subset of samples with sufficient information on disease presentation.

As reported elsewhere ${ }^{15,16}$, Delta and Delta-plus variants were associated with severe infections in Ghana. The worldwide dominance of Delta has been linked to the $\mathrm{P} 681 \mathrm{H} / \mathrm{R}$ substitutions ${ }^{3}$ as well as additional mutations in the viral RNA-dependent RNA-polymerase coding sequence. It is opined that these mutations enhance replication speed and significantly increase the viral load in infected persons ${ }^{17}$. As such, it was not surprising that Delta Plus accounted for over $50 \%$ of the severe cases of COVID-19 successfully sequenced in the current study. This is consistent with data from India, where Delta was first detected in clinical cases and, together with Delta-plus sub-lineages, were responsible for many COVID-19 fatalities in that country ${ }^{18,19}$. Indeed, Delta has been shown to increase COVID-19 severity and poor prognosis over 235 -fold in certain populations ${ }^{20}$. Unlike most previous variants, nearly all available vaccines have demonstrated limited efficacy against Delta and its sub-lineages ${ }^{21}$, but this trend may also be population-specific since other studies have shown high efficacy ${ }^{21,22}$. Despite very low vaccinations rates and the dominance of Delta and its sub-lineages, severe infections and Case Fatality Rates remain low to date, a further indication of the apparent resilience of the Ghanaian population to COVID-19 highlighted in our previous study ${ }^{5}$. Nevertheless, as a limitation to the study, the dominance of the VOC in our study may result from the purposive sampling and potential underrepresentation of some variants in other regions.

Through continuous genomic surveillance, we have characterised the diversity and evolution of COVID-19 variants in Ghana. We observed high variation in the number of mutations between samples, suggesting rapid evolution and multiple independent emergences of the Ghanaian variants against the backdrop of a multi-ethnic society. Besides, high mutation frequency within a population leads to higher chances of diverse variants. Although our study has the limitation of relying on purposive sampling, the large sample size and the depth of genetic analysis performed give high confidence that these data are robust and provide a credible overview of the evolution of the pandemic in Ghana. Apparent resilience to disease severity notwithstanding, this study indicates that Ghana is not impervious to the entry of novel variants

Page $11 / 24$ 
nor immune to their increased pathogenesis. Enhancing and speeding up vaccinations should be a priority, as well as the pursuit of therapeutic options. Ongoing virus and virus-host interaction experiments, combined with enhanced studies on the severe/critically ill patients, should also give more insights into the pathogenesis of SARS-CoV-2 in Ghana.

\section{Methods}

\section{Study Participants}

The study was approved by the Ethics Review Committee of Ghana Health Service (GHS-ERC 005/06/20), the Ethical Committee of College of Basic and Applied Sciences of the University of Ghana (ECBAS 063/19-20) and the Research Ethics Committee (REC) of the University of Health and Allied Sciences with certificate number UHAS-REC WV [1] 21-22. Samples were obtained from individuals reporting to community COVID-19 testing laboratories in different regions (Ashanti, Bono East, Central, Eastern, Greater Accra, Northern, Upper East, Volta (both Volta and Oti) and Western) of the country, and travellers who tested positive for COVID-19 on arrival at the Kotoka International Airport. Individuals tested at the community laboratories included; patients reporting to hospitals, individuals sampled during contact tracing, or those taking tests for travel purposes. The samples from passengers that arrived in Ghana by air were collected at three-time points (January 2021, March 2021, and June 2021), while the community samples were continuously collected from the testing laboratories from June 2020 to August 2021. Informed consent was obtained from patients directly or through Ghana Health Service authorization for samples obtained as part of routine surveillance. All samples were sequenced at West African Centre for Cell Biology of Infectious Pathogens (WACCBIP), University of Ghana, except some of the samples from Volta Region, which were collected and sequenced at the University of Health and Allied Sciences (UHAS) COVID-19 Testing and Research Centre.

\section{Sample selection and processing}

Samples confirmed as SARS-COV-2 positive by Real-Time PCR with cycle threshold (CT) values in the range 12-35 were selected for genome sequencing. Viral RNA from nasopharyngeal and oropharyngeal samples was extracted using the QIAmp Viral RNA extraction kit (Qiagen, Hilden, Germany). The extracted total RNA concentration was measured using Qubit ${ }^{\mathrm{TM}}$ RNA HS Assay Kit on a Qubit 4 Fluorometer (Thermo Fisher Scientific TM, MA USA). The integrity and quality of RNA were checked using the Agilent RNA 6000 Nano Kit on the Bioanalyzer (Agilent ${ }^{\mathrm{TM}}$ Tech. Inc. CA USA). The extracted RNA was immediately used for CDNA synthesis. The ARTIC V3 primer pools were used for multiplex tiled PCR to generate amplicons from the cDNA for sequencing as protocol ${ }^{23}$.

\section{Generation of SARS-CoV-2 Genomes}

Base-calling and demultiplexing of MinION Fast5 files were performed using Guppy (version 3.4.3 -5.0.7) according to the ARTIC bioinformatic protocols ${ }^{23}$. Sequencing QC was performed using pycoQC, and predemultiplexed reads were aggregated and length filtered using ARTIC guppyplex for a minimum of 400 
reads and a maximum of 700 reads to remove chimeric reads. Read QC was performed using NanoPlot before reading alignment, variant calling and consensus generations using ARTIC MinION software (ARTIC version1.2.1). Alignment metrics, amplicon coverage, variant annotation, and consensus assessment were performed using samtools, mosdepth, BCFtools, SnpEFF, and Quast according to the nfcore/viralrecon pipeline (version 2.2) ${ }^{24,25}$. Variant annotation, validation and quality assessment of the consensus sequence were performed using Viral Annotation DefineR (VADR) (version 1.1.3) ${ }^{26}$. Genomes that pass quality control were deposited on GISAID. Phylogenetic assignment of the consensus sequence to the globally named outbreak lineages was performed using Pangolin (Version 2.0) according to Rambaut, Holmes ${ }^{27}$. A coverage map was generated by comparing the Ghanaian SARS-CoV-2 genomes and the reference genome (Wuhan-Hu-1/2019) using Nextclade CLI (version 1.4.0). This webbased tool performs banded Smith-Waterman alignment with an affine gap-penalty ${ }^{28}$. Nextclade was also used to perform clade assignment and overall quality assessment of the genomes. Further analysis was executed in $\mathrm{R}$ (version 4.0.4)

\section{Phylogenetic Analysis}

Phylogenetic analysis of the SARS-CoV-2 genomes was performed using the Nextstrain pipelines ${ }^{28}$. Briefly, the nextstrian pipeline incorporates various quality control processes, including; validation of the clinical metadata, aligning sequences using nextalign to identify gaps compared to the SARS-CoV-2 reference genome (MN908747.3), and performing pangolin to assign lineages labels ${ }^{28}$. Next, $100 \mathrm{bp}$ from the start and $50 \mathrm{bp}$ at the end were masked, and the regions prone to sequencing errors (13402, 24389 and 24390) ${ }^{28}$. An initial maximum likelihood phylogenetic tree was constructed using augur's fast and stochastic algorithm (IQTREE) ${ }^{29}$ with a generalised time-reversible substitution model. This tree was refined to estimate divergence, time, and node dates using a coalescent timescale, then exported to auspice and R (version 4.0.4) for visualization. Molecular clock estimation for all the lineages and mutational fitness analysis for the B.1.1.318 lineage was also performed using nextstrain pipelines. The mutational fitness was based on hierarchical Bayesian multinomial logistic regression ${ }^{30}$.

\section{Data Availability}

The consensus sequences and basic metadata information for all the samples are freely available at GISAID (https://www.gisaid.org). The summary/analysis tables used to generate the piecharts/stacked plots are available at https://github.com/misita-falcon/SARS-CoV-2-Manuscript-2021.

\section{Code Availability}

The scripts used for the analysis reported in this study are publicly available at https://github.com/misita-falcon/SARS-CoV-2-Manuscript-2021.

\section{References}


1. WHO. WHO Coronavirus (COVID-19) Dashboard. (2021).

2. GHS. COVID-19 Situation Dashboard I Ghana - COVID-19 Updates.). Ghana Health Service (2021).

3. Tan C-W, et al. Pan-sarbecovirus neutralizing antibodies in BNT162b2-immunized SARS-CoV-1 survivors. New England Journal of Medicine 385, 1401-1406 (2021).

4. GHS. COVID-19: Ghana's Outbreak Response Management Updates. https://www.ghs.gov.gh/covid19/.). Ghana Health Services (2021).

5. Quashie PK, et al. Trends of severe acute respiratory syndrome coronavirus 2 (SARS-CoV-2) antibody prevalence in selected regions across Ghana. Wellcome Open Research 6, 173 (2021).

6. WHO. Tracking-SARS-CoV-2-variants.). World Health Organization (2021).

7. WHO. COVID-19 Weekly epidemiological February update.) (2021).

8. Zhao Y, Huang J, Zhang L, Chen S, Gao J, Jiao H. The global transmission of new coronavirus variants. Environmental research, 112240 (2021).

9. Ngoi JM, et al. Genomic analysis of SARS-CoV-2 reveals local viral evolution in Ghana. Exp Biol Med (Maywood) 246, 960-970 (2021).

10. Sy KTL, White LF, Nichols BE. Population density and basic reproductive number of COVID-19 across United States counties. PLoS One 16, e0249271 (2021).

11. Brown JC, et al. Increased transmission of SARS-CoV-2 lineage B. 1.1. 7 (VOC 2020212/01) is not accounted for by a replicative advantage in primary airway cells or antibody escape. BioRxiv, (2021).

12. Wilkinson $\mathrm{E}$, et al. A year of genomic surveillance reveals how the SARS-CoV-2 pandemic unfolded in Africa. Medrxiv, (2021).

13. Tegally $\mathrm{H}$, et al. Genomic epidemiology of SARS-CoV-2 in Mauritius reveals a new wave of infections dominated by the B. 1.1. 318, a variant under investigation. medRxiv, (2021).

14. Tao K, et al. The biological and clinical significance of emerging SARS-CoV-2 variants. Nat Rev Genet, 1-17 (2021).

15. Mishra S. How dangerous is the new Delta Plus variant? Here's what we know.). National Geographic (2021).

16. CDC. Delta Variant: What We Know About the Science COVID-19.) (2021).

17. Pachetti $\mathrm{M}$, et al. Emerging SARS-CoV-2 mutation hot spots include a novel RNA-dependent-RNA polymerase variant. Journal of translational medicine 18, 1-9 (2020).

18. Salvatore M, et al. Resurgence of SARS-CoV-2 in India: potential role of the B. 1.617. 2 (delta) variant and delayed interventions. medRxiv, (2021).

19. Joshi M, et al. First detection of SARS-CoV-2 Delta variant (B. 1.617. 2) in the wastewater of (Ahmedabad), India. medRxiv, (2021).

20. Fisman DN, Tuite AR. Evaluation of the relative virulence of novel SARS-CoV-2 variants: a retrospective cohort study in Ontario, Canada. CMAJ: Canadian Medical Association Journal 193, E1619 (2021). 
21. Lopez Bernal J, et al. Effectiveness of Covid-19 vaccines against the B. 1.617. 2 (Delta) variant. $N$ Engl J Med, 585-594 (2021).

22. Tang $P$, et al. BNT162b2 and mRNA-1273 COVID-19 vaccine effectiveness against the SARS-CoV-2 Delta variant in Qatar. Nature Medicine, 1-8 (2021).

23. Wick RR, Judd LM, Holt KE. Performance of neural network basecalling tools for Oxford Nanopore sequencing. Genome biology 20, 1-10 (2019).

24. Ewels PA, et al. The nf-core framework for community-curated bioinformatics pipelines. Nature biotechnology 38, 276-278 (2020).

25. U. Garcia; jcurado; Kevin Menden HPSVSMJE-CMLHGGn-cbPEMSKKSM. nf-core/viralrecon: nfcore/viralrecon v2.2 - Tin Turtle.) (2021).

26. Schäffer $A A$, et al. VADR: validation and annotation of virus sequence submissions to GenBank. $B M C$ bioinformatics 21, 1-23 (2020).

27. Rambaut A, et al. A dynamic nomenclature proposal for SARS-CoV-2 lineages to assist genomic epidemiology. Nature microbiology 5, 1403-1407 (2020).

28. Hadfield J, et al. Nextstrain: real-time tracking of pathogen evolution. Bioinformatics $34,4121-4123$ (2018).

29. Nguyen L-T, Schmidt HA, Von Haeseler A, Minh BQ. IQ-TREE: a fast and effective stochastic algorithm for estimating maximum-likelihood phylogenies. Molecular biology and evolution 32, 268274 (2015).

30. Obermeyer FH, et al. Analysis of 2.1 million SARS-CoV-2 genomes identifies mutations associated with transmissibility. medRxiv, (2021).

\section{Declarations}

Acknowledgements

The study was facilitated by the Government of Ghana through the Ghana Health Service. ARTIC donated the primers used for the study. All data storage and analyses were performed on Zuputo $®$, the University of Ghana's high-performance computing cluster. We acknowledge the Community and Public engagement members; Kyerewaa A. Boateng and Simon Donkor, Andrew M. Nantogmah and the entire WACCBIP and UHAS COVID-19 Teams.

Funding

The study was funded by a grant from the Rockefeller Foundation (2021 HTH 006), an Institut de Recherche pour le Développement (IRD) grant (ARIACOV), African Research Universities Alliance (ARUA) Vaccine Development Hubs grant with funds from Open Society Foundation, a Wellcome/African Academy of Sciences Developing Excellence in Leader'ship Training and Science (DELTAS) grant (DEL-15007 and 107755/Z/15/Z: Awandare); National Institute of Health Research (NIHR) (17.63.91) grants using UK aid from the UK Government for a global health research group for Genomic surveillance of 
malaria in West Africa (Wellcome Sanger Institute, UK) and global research unit for Tackling Infections to Benefit Africa (TIBA partnership, University of Edinburgh); and the World Bank African Centres of Excellent grant (WACCBIP-NCDs: Awandare). CMM and DSYA are supported by WACCBIP DELTAS PhD fellowships, while PQ, and YB are supported by a Crick African Network Career Accelerator fellowship. The University of Health and Allied Sciences, COVID-19 Testing and Research Centre was supported by China Novartis Institutes of Biomedical Research (CNIBR-SCD: Duedu) and the Grand Challenges Africa programme grant GCA/AMR/rnd2/138: Duedu). The views expressed in this publication are those of the author(s) and not necessarily those of the funders.

Author Information

Affiliations:

West African Centre for Cell Biology of Infectious Pathogens (WACCBIP), University of Ghana, Accra, Ghana

Collins M. Morang'a, Joyce M. Ngoi, Dominic S. Y. Amuzu, Philip M. Soglo, Vincent Appiah, Violette V. M'cormack, Frederick Tei-Maya, Evelyn B. Quansah, Enock K. Amoako, Isaac T. Ogbe, Bright K. Yemi, Israel Osei-Wusu, Deborah N. A. Mettle, Samirah Saiid, Kesego Tapela, Francis Dzabeng, Jerry Quaye, Precious C. Opurum, Yaw Bediako, Peter K. Quashie, Lucas N. Amenga-Etego, Gordon A. Awandare

University of Health and Allied Sciences COVID-19 Testing and Research Centre, Ho, Ghana Jones Gyamfi, Reuben Ayivor-Djanie, Rosina A. Carr, John O. Gyapong, Kwabena O. Duedu

Tamale Teaching Hospital Intensive Care Unit, Ghana Health Service, Tamale, Ghana

Benjamin D. Nuertey, Dam K. Mibut

Tamale Public Health and Reference Laboratory, Ghana Health Service, Tamale, Ghana Abdul-Karim Abass

Noguchi Memorial Institute for Medical Research, University of Ghana, Legon, Accra, Ghana Vanessa Magnussen, Ivy A. Asante, William K. Ampofo

\section{LEDing Medical Laboratory, Accra, Ghana}

Paul Owusu-Oduro

Ghana Infectious Disease Centre, Accra, Ghana

Joseph Oliver-Commey 
Kintampo Health Research Centre, Research and Development Division, Ghana Health Service, Kintampo North Municipality, Ghana

Dennis Adu-Gyasi, Nicholas Amoako, Kwaku Poku Asante

Biomedical and Public Health Research Unit, Council for Scientific and Industrial Research, Accra, Ghana

Samuel Armoo, Mike Y. Osei-Atweneboana

Kumasi Centre for Collaborative Research in Tropical Medicine, Kwame Nkrumah University of Science and Technology, Kumasi, Ghana

Michael Owusu, Augustina Sylverken, Richard O. Phillips

Institutional Care Division (ICD), Ghana Health Service, Accra, Ghana

Samuel K. Akoriyea

Accra Veterinary Laboratory, Veterinary Services Directorate, Accra, Ghana

Edward D. Fenteng, Patrick Tetteh Ababio

Cape Coast Teaching Hospital, Ghana Health Service, Cape Coast, Ghana

Emmanuella Amoako, Frederick Kumi-Ansah

Takoradi Veterinary Services Department, Ghana Health Service, Takoradi, Ghana

Oliver D. Boakye, Theophilus Odoom

Ga East Municipal Hospital, Ghana Health Service, Accra, Ghana

Lawrence Ofori-Boadu

Pong-Tamale Central Veterinary Laboratory, National Veterinary Services Directorate, Tamale, Ghana

Emmanuel Allegye-Cudjoe

Navrongo Health Research Centre, Research and Development Division, Ghana Health

Service, Navrongo, Ghana

Sylvester Dassah, Victor Asoala

Ghana Health Service, Accra, Ghana

Patrick Kuma-Aboagye 


\section{Institut de Recherche pour le Développement, Marseille, France}

Nicaise T. Ndam

\section{Yemaachi Biotechnology, Accra, Ghana}

Yaw Bediako

Contributions

GAA, PKQ, YB, and KOD conceived the study. GAA, KOD, and NTN obtained the funding, and GAA, PKA, JOG and WKA supervised the work. PKQ, KOD and LNA supervised the sequencing and data analyses. JMN performed the viral RNA extraction and sequencing along with FT-M, EBQ, BKY, DNAM, IOW, SS, VM, KT, JQ, PCO, JG and RAC. BDN, IAA, POO, SA, DAG, NA, JOC, MO, AS, EDF, RAD, PTA, AKA, SKA, EA, FKA, ODB, DKM, TO, LOB, EAC, SD, VA, KPA, ROP, MYOA, and PKA contributed to the detection and selection of SARS-CoV-2 positive samples for sequencing. Sequence analysis and generation of figures was performed by CMM, DSYA, VA, EKA, PMS, FD, VVM and ITO. DSYA, CMM, JMN, PMS and VVM drafted the manuscript. LNA, PKQ and GAA critically reviewed and edited the manuscript. All authors read and approved the final version of the manuscript.

\section{\#Corresponding authors}

Gordon A. Awandare,

gawandare@ug.edu.gh,

Lucas N. Amenga-Etego

lamenga-etego@ug.edu.gh

Peter K. Quashie

pquashie@ug.edu.gh

\section{Ethics Declarations}

Competing Interests

The author(s) declared no potential conflicts of interest concerning the research, authorship, and publication of this article.

\section{Figures}




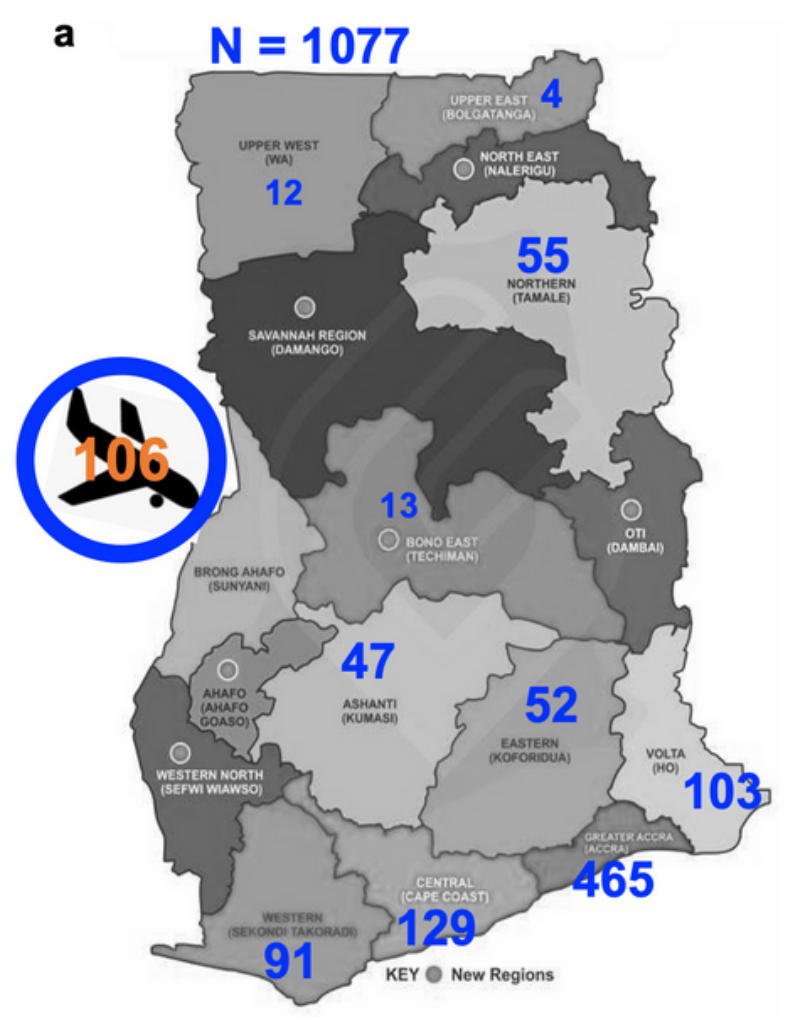

b

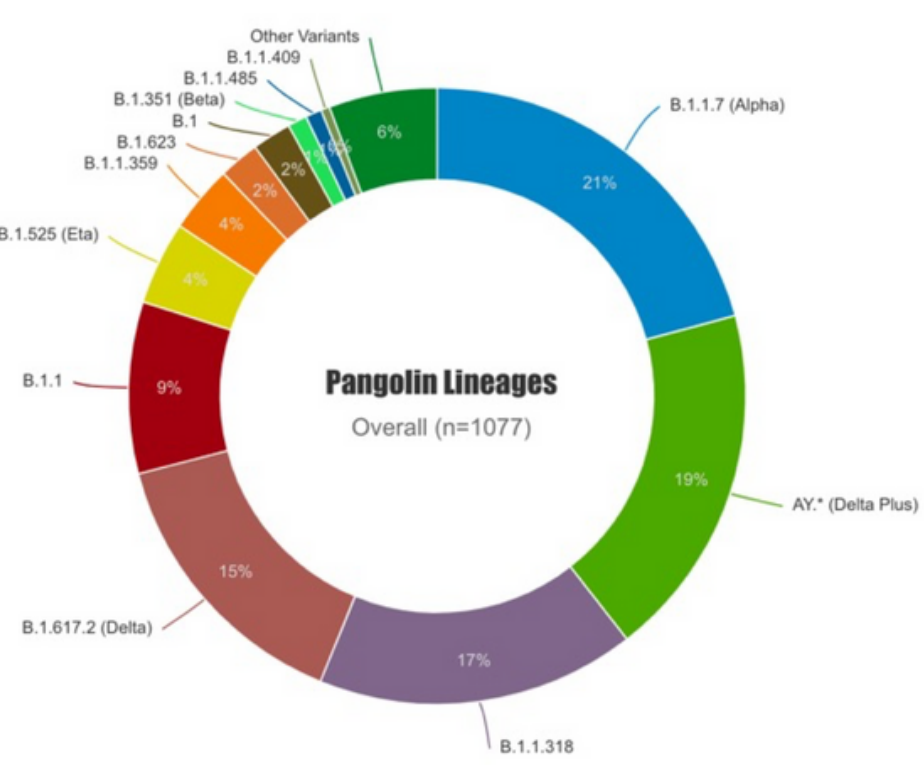

C

\section{d}

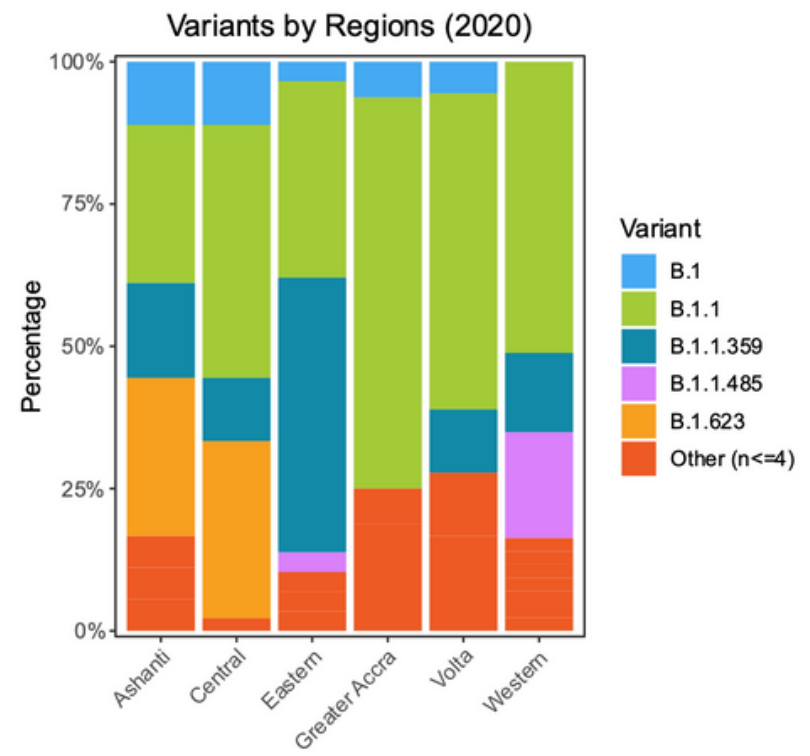

Regions

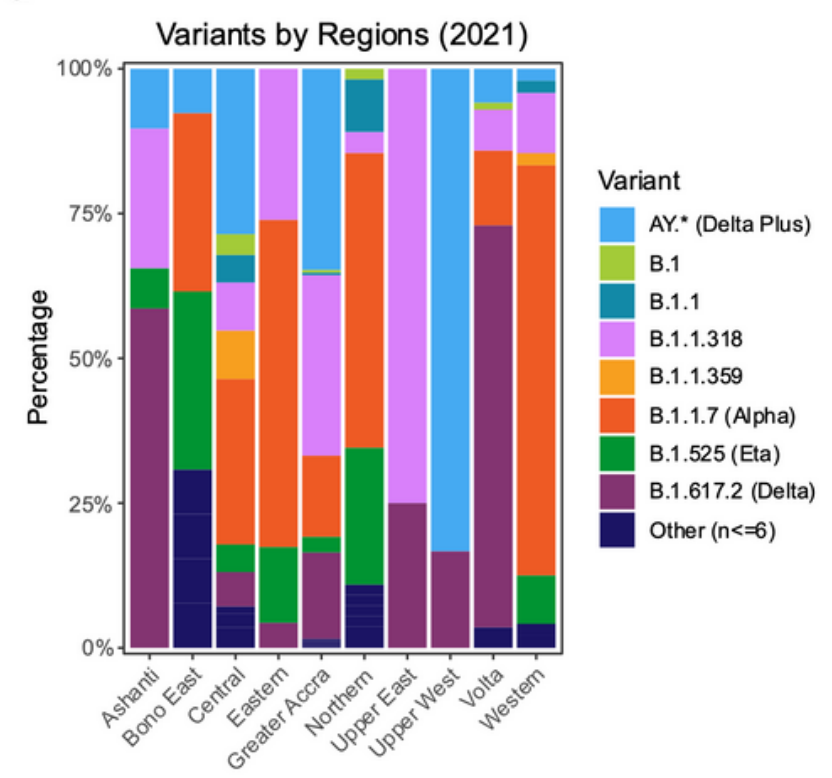

Regions

\section{Figure 1}

Overall genomic epidemiology of the circulating variants across various regions in Ghana. a) Map showing the ten sampled regions ( $n=971 / 1077$ and travellers arriving in the country (106/1077) b) SARSCoV-2 lineages in Ghana; Alpha, (B.1.1.7) variant (blue), Delta Plus (green), B.1.1.318 (purple) and Delta (brown). c) Lineage proportions within the local Ghanaian isolates in $2020(n=169)$ d) Lineage proportions within the local Ghanaian isolates in 2021 ( $n=802)$. 
a

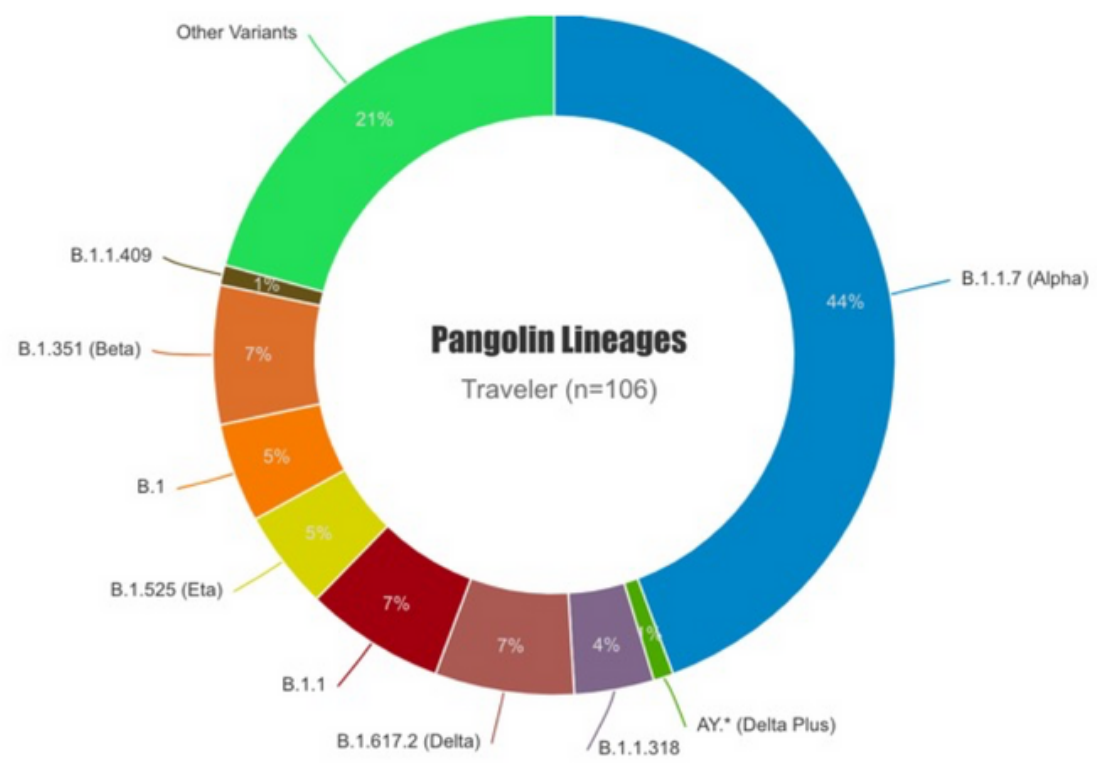

C

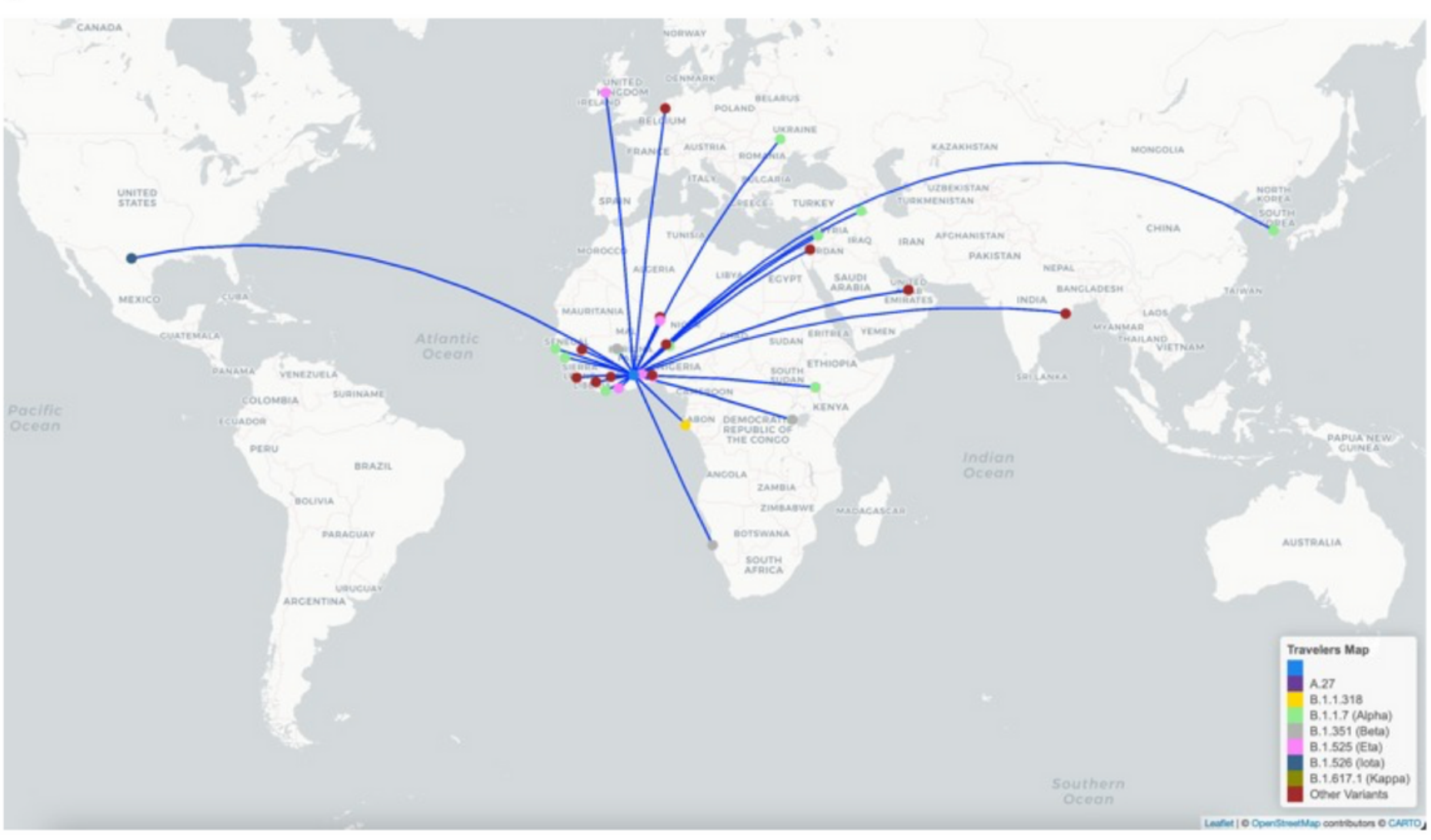

\section{Figure 2}

Transmission of SARS-CoV-2 variants into Ghana from other countries $(n=106)$. a) Fre-quency of SARSCoV-2 variants among travellers arriving in Ghana. b) The proportion of vari-ants among travellers arriving in Ghana in January, March, and June. c) The map indicates the different countries each traveller came from into Ghana. The coloured circles represent the in-dividuals carrying the respective variants (coded by colour) from the origin of travel and arriv-ing in Ghana. 
a

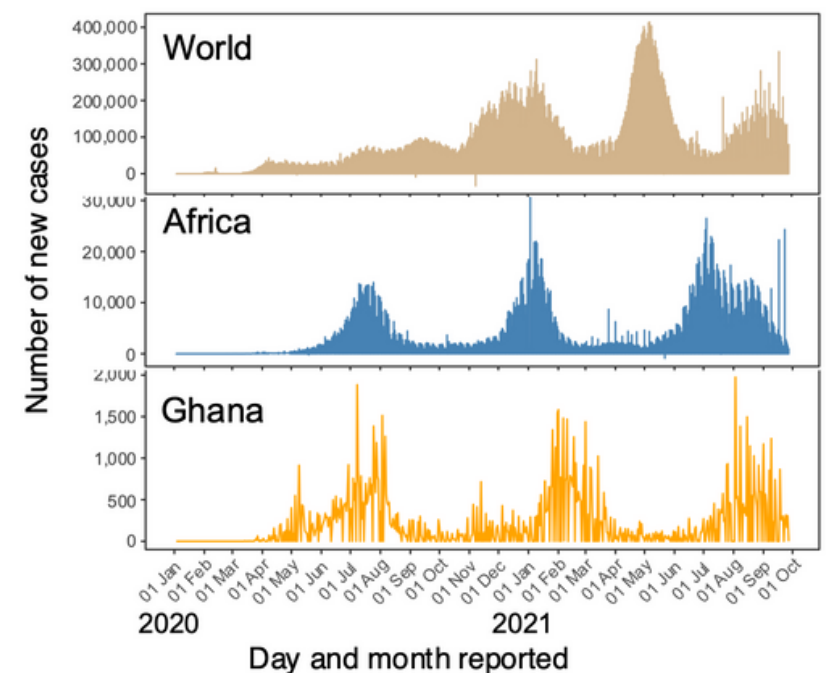

C

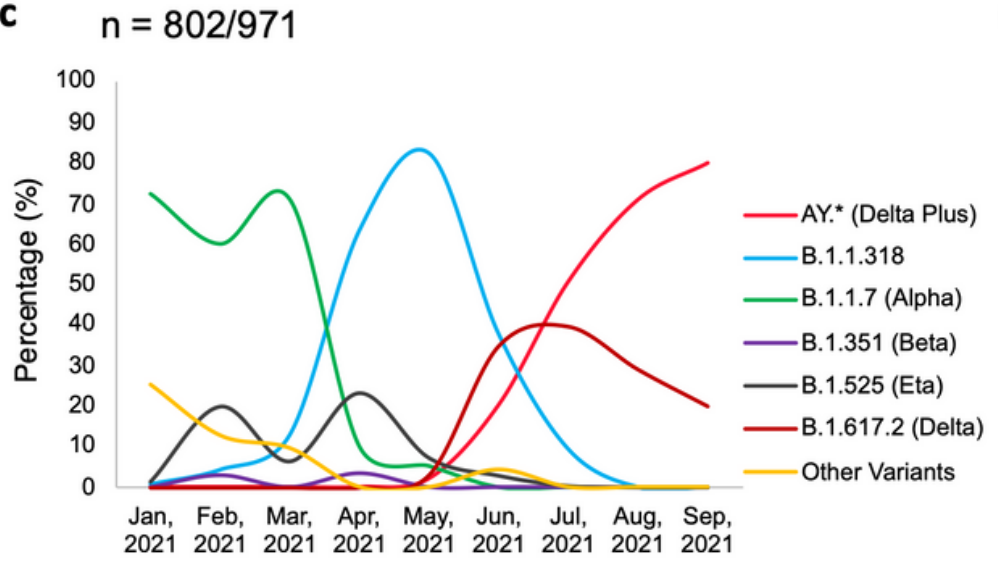

Month, Year

\section{b}

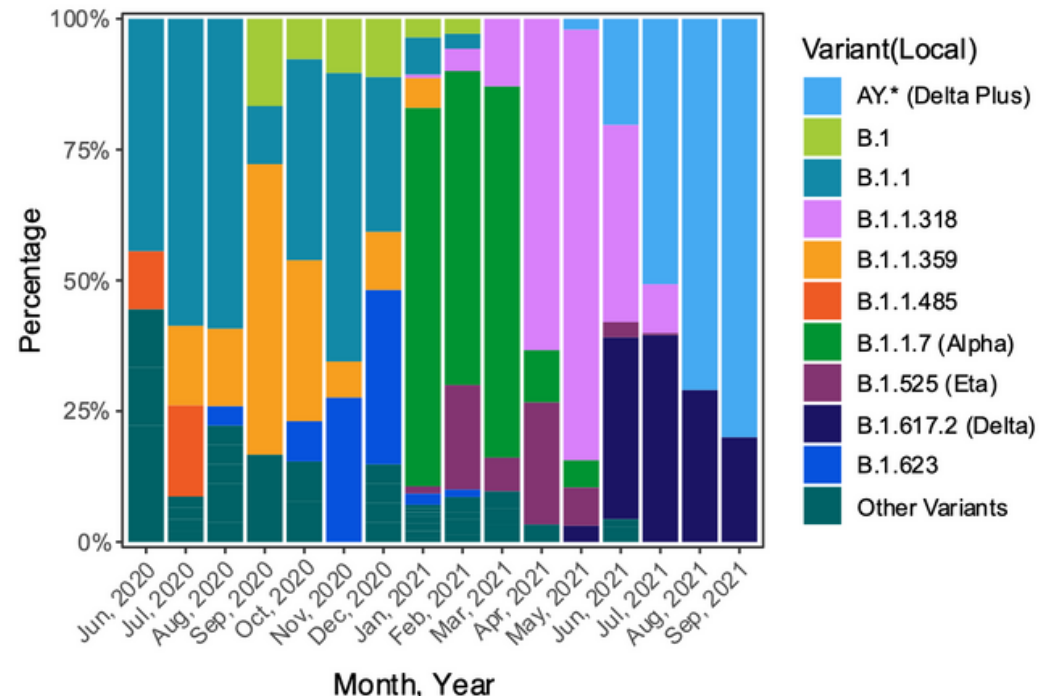

d

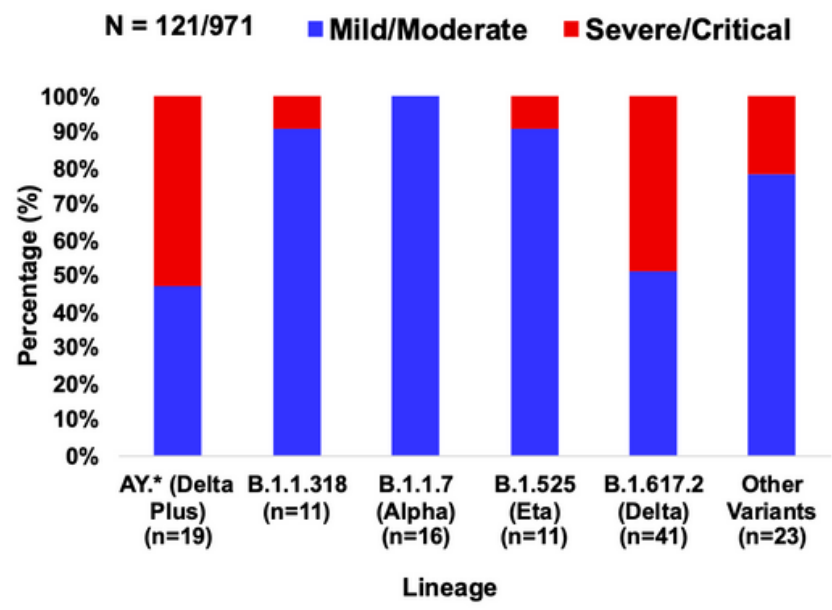

\section{Figure 3}

Trends in the variant epidemiology in Ghana. a) Cases of COVD-19 in Africa and Ghana, which indicates months mapped against new cases. This shows the number of confirmed COVID-19 cases recorded daily in World (tan), Africa (blue) and Ghana (gold) from January 2020 to October 2021. The data was obtained from WHO (WHO, 2021) b) Trends in prevalence of major variants circulating in Ghana from June 2020 to September 2021 ( $n=971 / 1077)$. c) Im-pact of emergence of variants of concern (VOCs) on dynamics of viral lineages dominating community transmission in Ghana in 2021. d) Disease Severity associated with the VOC; line-ages mapped against percentage distribution of clinical presentation of SARS-CoV-2, including mild/moderate (blue) or severe/critical (orange) ( $n=121 / 971)$. 
a

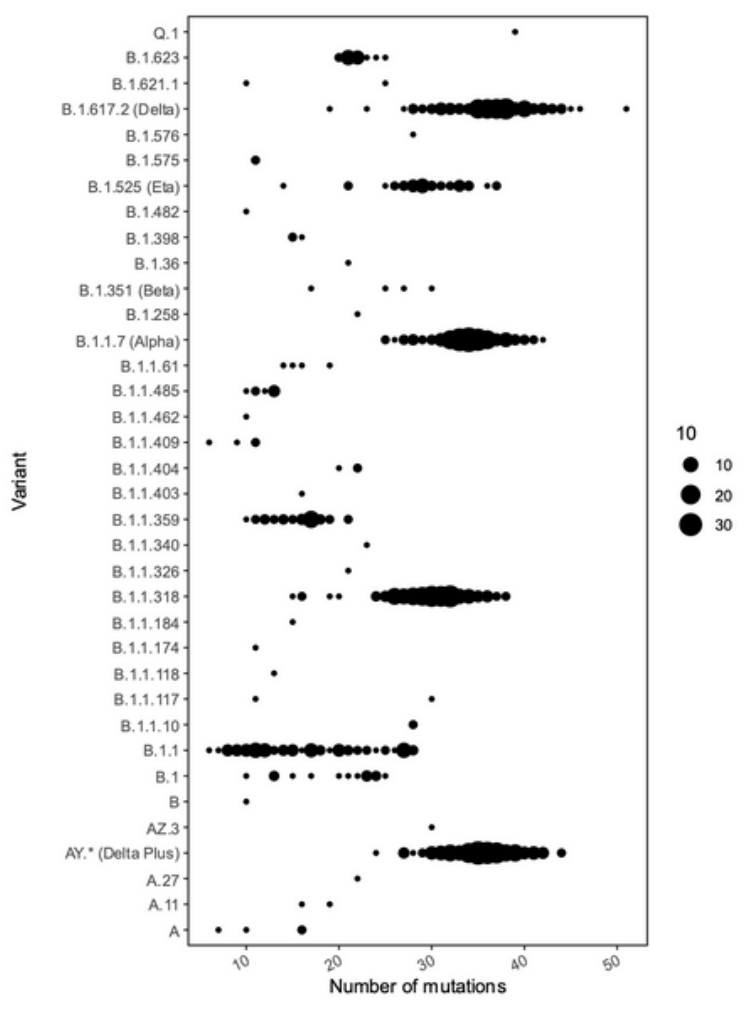

b

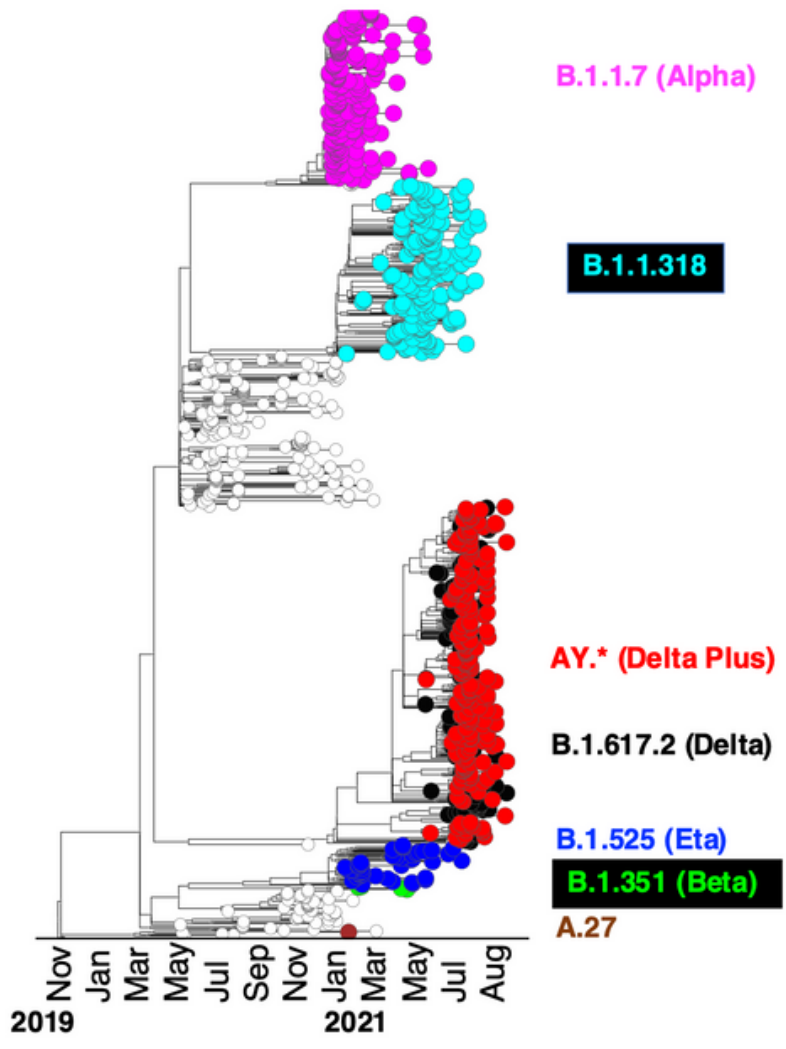

d

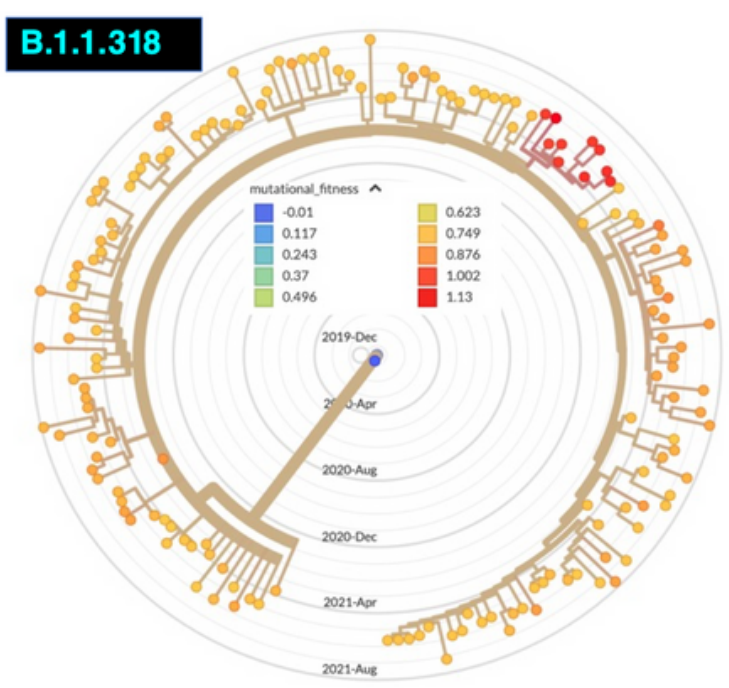

\section{Figure 4}

Genetic diversity and molecular evolutionary relationships of variants identified in Gha-na. a) The spread/range and magnitude of mutation per lineage $(n=971)$. Each filled circle rep-resents a sample, and the circle's width is proportional to the number of mutations present in a particular sample. b) Maximum likelihood phylogenetic tree with ancestral state reconstruction in a backdrop of reference sequences from Wuhan and evolutionary relationship of the Ghana-ian variants over time $(n=971)$. Colours show the 
VOC; Alpha (purple), B.1.1.318 (cyan), Beta (green), Eta (blue), Delta (black) and Delta plus (red). c) Rootto-tip divergence as a function of sampling time. The $Y$-axis denoted divergence (the number of mutations in the genome rela-tive to the root), and the $\mathrm{X}$-axis shows the sampling date of each genome [Alpha (lapis), B.1.1.318 (royal blue), B.1.351 (azure blue), Eta (cyan) and Delta (apple)]. d) Annotated muta-tional fitness of all the B.1.1.318 lineage; the samples with the highest fitness are coloured red.

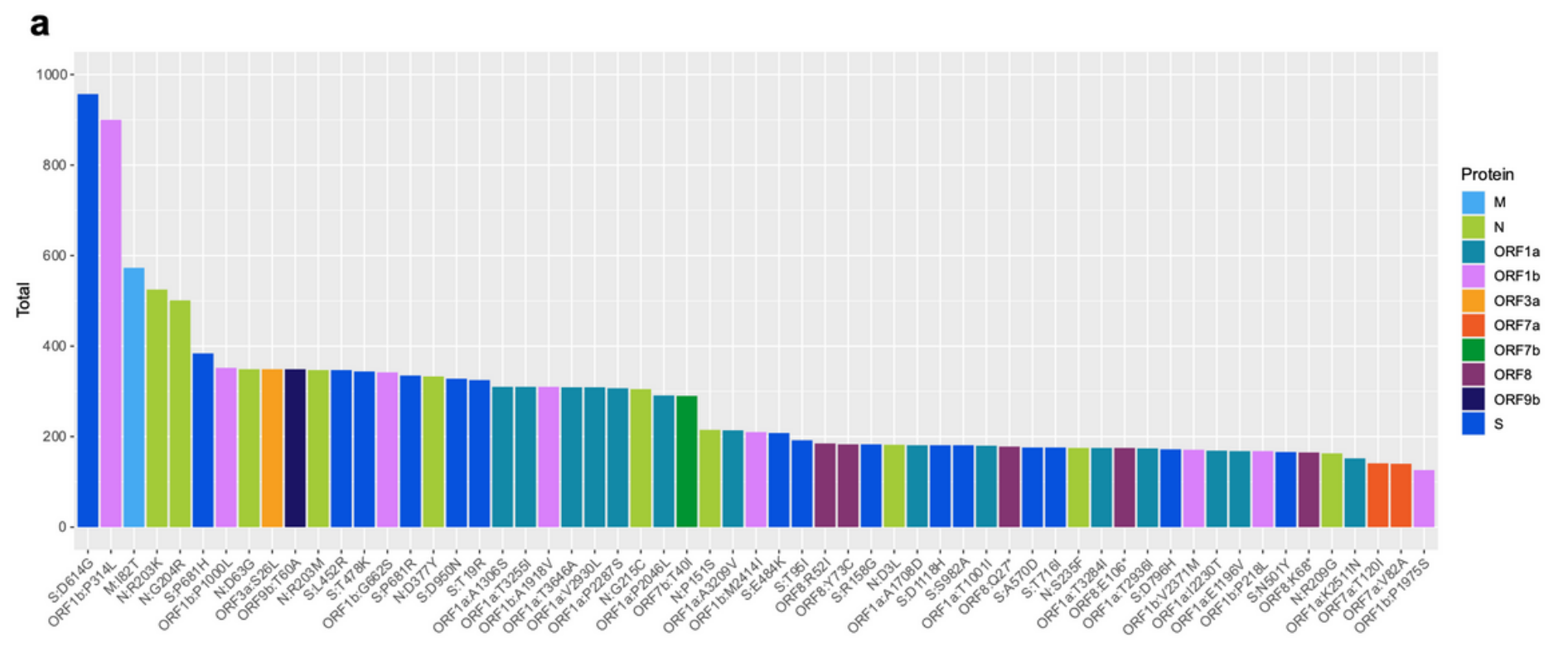

b

\section{Genome-wide amino acid substitutions}

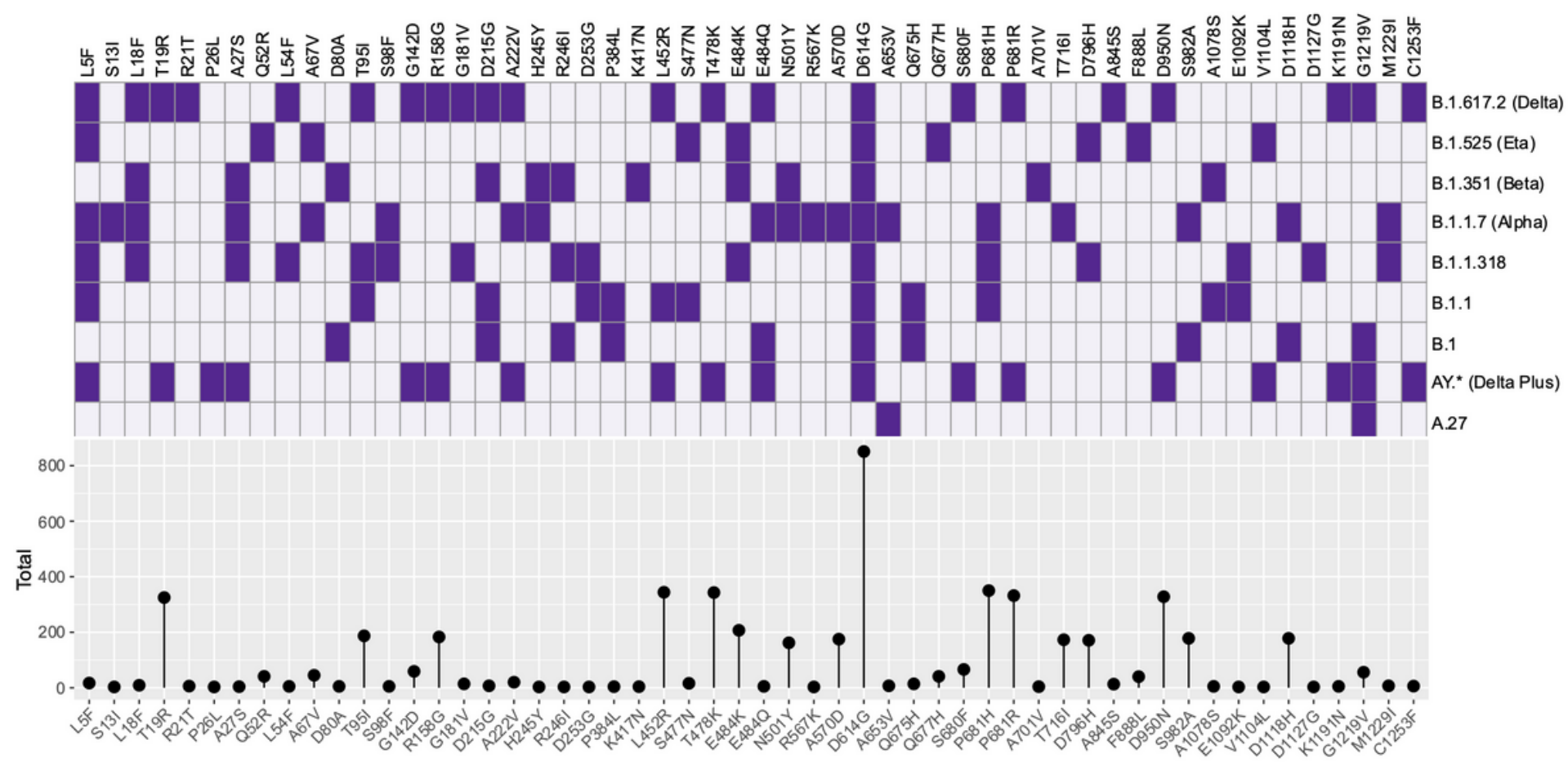

\section{Spike protein amino acid substitutions}

\section{Figure 5}

Analysis of single nucleotide polymorphisms (SNPs) in variants circulating in Ghana. a). Frequencies of amino acid substitutions across all the SARS-CoV-2 proteins in all genomes sequenced b) Spike 
glycoprotein amino-acid substitutions present/absent in major SARS-CoV-2 variants circulating in Ghana. Purple shading indicates mutations within the lineages, where-as white indicates the absence of the mutation in a particular variant in all the samples. The bottom panel plot shows the frequencies of individual mutations across all the samples.

\section{Supplementary Files}

This is a list of supplementary files associated with this preprint. Click to download.

- ReportingSummaryNCOMMS2145494GeneticDiversityofSARSCoV22021.pdf 